\title{
Parameterization of Debye Model for Dielectrics Using Voltage Response Measurements and a Benchmark Problem
}

\author{
Ehtasham Mustafa1,2, Regina Mária Németh², Ramy S. A. Afia ${ }^{2,3}$, Zoltán Ádám Tamus²* \\ 1 Department of Electrical Engineering, Faculty of Engineering \& Technology, Gomal University, Main Multan Road, 29050 Dera \\ Ismail Khan, Kyber Pakhtunkhwa, Pakistan \\ 2 Department of Electric Power Engineering, Faculty of Electrical Engineering and Informatics, Budapest University of \\ Engineering and Economics, 18. Egry József str., H-1111 Budapest, Hungary \\ ${ }^{3}$ Department of Electrical Power and Machines Engineering, Faculty of Engineering, Helwan University, 1 Sherif Street, \\ 11792 Helwan, Cairo, Egypt \\ *Corresponding author, e-mail: tamus.adam@vet.bme.hu
}

Received: 07 May 2020, Accepted: 03 September 2020, Published online: 16 April 2021

\begin{abstract}
The Voltage Response measurement since its introduction in the 1960s has been used successfully for the diagnostics of electrical insulation. The method is based on two quantities of decay and return voltage slopes and can be used to study the conduction and polarization processes inside the insulation. Extended Voltage Response method, being an advanced version of the Voltage Response measurement helps in further studying the polarization process by using a large polarization spectrum and hence dielectric relaxation processes. These dielectric relaxation processes can be modeled by the Debye model. Since as most of the techniques used for diagnostic purpose does not give the information about the conduction and polarization processes separately, it is difficult to determine the $R$ - $C$ parameters of the Debye model. The Voltage Response technique is very useful in this regard because of the two voltage slopes. The paper shows a novel experimental benchmark for testing the function fitting methodologies of the Voltage Response methodologies, which helps in determining the $R$-C parameters. Moreover, the problem can be used for testing the novel genetic, evolutionary algorithms, where benchmarking is an actual challenge. The proposed nonlinear function fitting method uses the genetic algorithms via the Ârtap framework, which lets it possible to select the most accurate optimization algorithm from the provided list of the algorithms and achieve better fitting precision, faster calculation time or more powerful processing ability.
\end{abstract}

Keywords

Voltage Response (VR), Extended Voltage Response (EVR), Debye model, optimization, Ārtap framework, benchmark problem

\section{Introduction}

Aging of electrical insulating materials has been a topic of great interest for many years, which increased the importance of diagnostic techniques to determine the condition of electrical insulations $[1,2]$. The measurement of the dielectric properties is a most important tool among the diagnostic techniques. The dielectric properties can be measured either using a time-domain method or frequency domain method [3]. Out of numerous time-domain techniques, measurement of return voltage is one such method, which was introduced in the 1960's by Endre Németh [4]. The concept behind the technique was to study the slow dielectric polarization processes inside the insulation material. Two techniques emerged from the voltage return method, Return Voltage Measurement (RVM) and Voltage Response (VR) method.
The non-destructive nature of the techniques gained the attention of the researcher for insulation diagnosis. Since then, the techniques have been used by many researchers for a wide range of electrical insulations to study the aging and the phenomenon of dielectric relaxation processes in the insulations [4-6]. Apart from its simplicity and robustness, the technique has a disadvantage of having a long measurement time [7]. In recent times, an extended version of the VR method Extended Voltage Response (EVR) method was introduced, which is useful to study in detail the polarization processes by measuring more than one return voltage slopes achieving a polarization spectrum [5-8].

It is well established that the dielectric can be modeled using the extended Debye circuit having $R$ and $C$ elements, 
which are used for model the conduction and polarization phenomenon of insulation (see Fig. 1). In this circuit model the $R_{0}$ represents the dc conductivity, the $C_{0}$ is the capacitiy of the electrode arrangement without dielectric. The $R_{p i}-C_{p i}$ branches (Debye elements) represent the elementary polarisaion processes of the tested insulation. The determination of parameters of Debye branches can help to identify the characteristic ageing processes of insulations. Understanig the ageing phenomenon is essential for reliable life-time management of electrical equipment.

Besides the conventional iteration methods used to determine the parameters of extended Debye model, in this research work, a novel function fitting methodologies based on experimental benchmark problem solving is used [9-12]. A physical Debye circuit insulation models were used for experiments and the Voltage Responses of the models were measured. Then based on the Voltage Response measurement results of insulation models, a nonlinear function fitting method using genetic algorithms via the Ârtap framework is adopted. This helps in the selection of the most accurate optimization algorithm from the provided list of the algorithms [13, 14]. The results show that the technique was helpful in the computationally hard problem to calculate the $R$ and $C$ parameters of the Debye model due to its powerful processing ability and faster calculation speed. Moreover, this method finds the global optimum of the problem.

\section{Extended Voltage Response measurements}

The Extended Voltage Response is a developed method of Voltage Response measurement [4-6]. The measurement of Voltage Response is based on the measurement of decay and return voltages of a charged insulation. The test arrangement can be seen in Fig. 2 and the timing diagram of the measurement is in Fig. 3.

For charging, the SW1 is in "ON" position. The SW2 is used for discharging the charged insulation. During the measurement of Voltage Responses both switcheas are

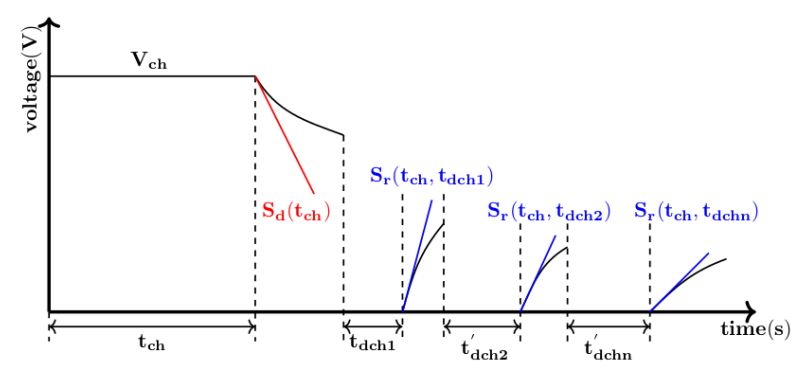

Fig. $1 R$ - $C$ equivalent circuit of insulation based on extended Debye model

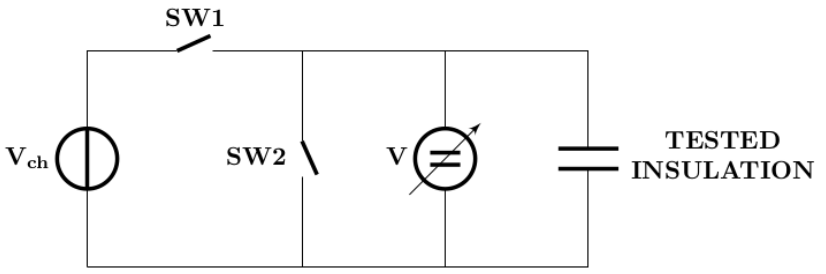

Fig. 2 Arragement of Voltage Response measurement

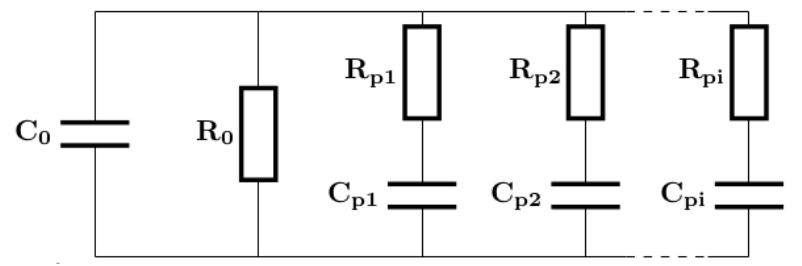

Fig. 3 The timing diagram of Extended Voltage Response measurement

in "OFF" position. The decay and return voltages are characterized by their intital slopes namely $S_{d}\left(t_{c h}\right)$ and $S_{r}\left(t_{c h}, t_{d c h n}\right)$, repectively (see Fig. 3). Obviously these values are dependent on the charging $\left(t_{c h}\right)$ af discharging times $\left(t_{d c h n}\right)$.

The values of Debye branches can be calculated by the initial slopes of return voltages. The voltage of a $C_{p i}$ capacitor $\left(V_{C_{p i}}\right)$ after tch charging and tdchn discharging times can be calculated by the multiplication of the exponential functions.

$V_{C_{p i}}=V_{c h}\left(1-e^{-t_{c h} / \tau}\right) \times e^{-t_{c c h} / \tau}$

Where $V_{c h}$ is the charging voltage and $\tau=R_{p i} C_{p i}$. The slope of return voltage $S_{r i}\left(t_{c h}, t_{d c h n}\right)$ of one Debye element (i.e. one $R_{p i}-C_{p i}$ branch) can be calculated by

$S_{r i}\left(t_{c h}, t_{d c h n}\right)=\frac{V_{c h}\left(1-e^{-t_{c h} / \tau}\right) \times e^{-t_{d c h} / \tau}}{R_{p i} C_{0}}$.

If the equivalent circuit contains $N$ Debye element, the total slope of return voltage can be cvalculated by using a superposition of return voltage slopes of each Debye element:

$S_{r}\left(t_{c h}, t_{d c h n}\right)=\sum_{i=1}^{N} S_{r i}\left(t_{c h}, t_{d c h n}\right)$

The determination of the values of Debye elements is based on the calculations above.

\section{Nonlinear curve fitting with Ārtap}

Curve fitting is a very general and important problem in science and industry. According to the type of the fitted function it can be categorized as a linear and non-linear optimization task. The literature contains a lot of different methods to solve this problem with the required accuracy [15-26]. 
Most of these measurements use the gradient-based solvers, the Gauss-Newton Algorithm or the LevenbergMarquardt Algorithm, which use the fact that these problems can be expressed by the rules of the Quadratic Programming (QP) [27, 28].

These methods use the fact that after the measurements, we have $m$ data points $t_{i} \in \mathbb{R}^{n} x \mathbb{R}$, where the least-squares fitting objective is

$$
\min _{\beta} \sum_{i=1}^{m}\left(f\left(x_{i} ; \beta\right)-y_{i}^{2}\right)=r_{1}^{2}+r_{2}^{2}+\ldots+r_{m}^{2},
$$

where $f$ is an instance of a convex function, $\beta \in \mathbb{R}^{n}$ is a vector, which contains the optimized parameters for the chosen function and $Y$ is a vector, which represents the measured values. The vector of the residuals $\boldsymbol{r}(\beta)$ can be defined in the following way:

$\boldsymbol{r}(\beta)=f(X ; \beta)-Y$,

where the task is to minimize the $\boldsymbol{r}(\beta)^{T} \boldsymbol{r}(\beta)$. Let the initial parameter is $\beta_{0}$, the value of the corresponding residual is $\boldsymbol{r}\left(\beta_{0}\right)$ and $\delta$ represent a small change in the input parameters, the new residual is

$\boldsymbol{r}\left(\beta_{0}+\delta\right) \approx \boldsymbol{r}\left(\beta_{0}\right)+\boldsymbol{J} \delta$,

where $\boldsymbol{J}\left(\in \mathbb{R}^{m \times n}\right)$ is the Jacobian of $f(\partial f / \partial \beta)$. From this, the problem can be expressed by its first order quadratic form in the following convex, quadratic form [26, 28]:

$$
\min _{\delta} \delta^{T} \boldsymbol{J}^{T} \boldsymbol{J} \delta+2 \delta^{T} \boldsymbol{J}^{T} \boldsymbol{r}+\boldsymbol{r}^{T} \boldsymbol{r}
$$

subject to

$\delta \in \Delta$,

where $\Delta$ is the trust region.

This task has a solution, when $\boldsymbol{r}(\beta)^{T} \boldsymbol{r}(\beta)=0$. The optimal step of the solution can be calculated by Gauss-Newton methods, which finds the $\delta$ that minimizes the quadratic objective, but with no trust-region bounds:

$$
\boldsymbol{J}^{T} \boldsymbol{J} \delta=-\boldsymbol{J}^{T} \boldsymbol{r} .
$$

The Levenberg-Marquardt algorithms can be used to solve this problem with trust regions:

$$
\boldsymbol{J}^{T} \boldsymbol{J}+\lambda \operatorname{diag}\left(\boldsymbol{J}^{T} \boldsymbol{J}\right) \delta=\boldsymbol{J}^{T} \boldsymbol{r},
$$

where $\lambda$ controls the magnitude of the quadratic penalty on $\delta$.

This methodology is widely used for linear and non-linear data-fitting. However, these algorithms are highly dependent on proper initial values, in the case of modeling engineering problems, correctly specifying the initial values is a hard task in the case of a practical problem [24]. Moreover, this approximated or interpolated functions have to be fitted on a noisy measurement data. One of the most widely used functions is the $b$-splines to describe a mathematical connection on the measured data [21]. Where the placement of the knots has to be optimized to minimize the error. This problem is a multi-modal and multi-variate nonlinear optimization problem with many local optima. Where the above mentioned gradient-based optimization methods can easily be trapped in a local optimum, because the optimization task cannot formulated as a convex optimization task. To overcome this problem there are many heuristics and metaheuristics developed for engineering applications. These methods are usually mixing some convex or mathematical optimization with a method of a branch and bound, genetic algorithms, neural networks or other meta-heuristics [17-20, 22-26, 29-34].

In this paper, a sum of exponentials has to be fitted. This task is highly non-linear, which complexity growth with the number of degrees of freedom ( $n$ is high). The size of the search space of the optimization task is large. Besides, the data is generally scattered and contains some noise from the measurement. A simple genetic algorithm-based fitting approach is used in this paper, via the Ārtap framework [9-12]. The main advantage of using this framework is the simplicity of the interface, which provides automatic parallelization and enables to exchange the applied genetic algorithm with another one. It is an important feature, because of the "no free lunch" theorem of optimization [34], which says that non-exists of an evolutionary methodology, which can overturn any other one. The pseudo-code of the NSGA-II algorithm is shown in Algorithm 1. NSGA-II is one of the most popularly used, a genetic algorithm-based, multi-objective optimization techniques [35-37]. Due to its three advantageous characteristics, which were outperformed the existing algorithms when it was published [38]. These properties are the elitism, the small computational complexity, which is almost $O\left(M N^{2}\right)$ and the explicit diversity preservation mechanism, which ensures good convergence and stability.

The object function can be defined in several ways, this type of optimization functions [19]:

$$
\begin{aligned}
& \text { - } F_{L S}=\sum_{i=1}^{n}\left(P_{i}-M_{i}\right)^{2} \\
& \text { - } F_{M E}=\sum_{i=1}^{n}\left(P_{i}-M_{i}\right)^{2} / n
\end{aligned}
$$




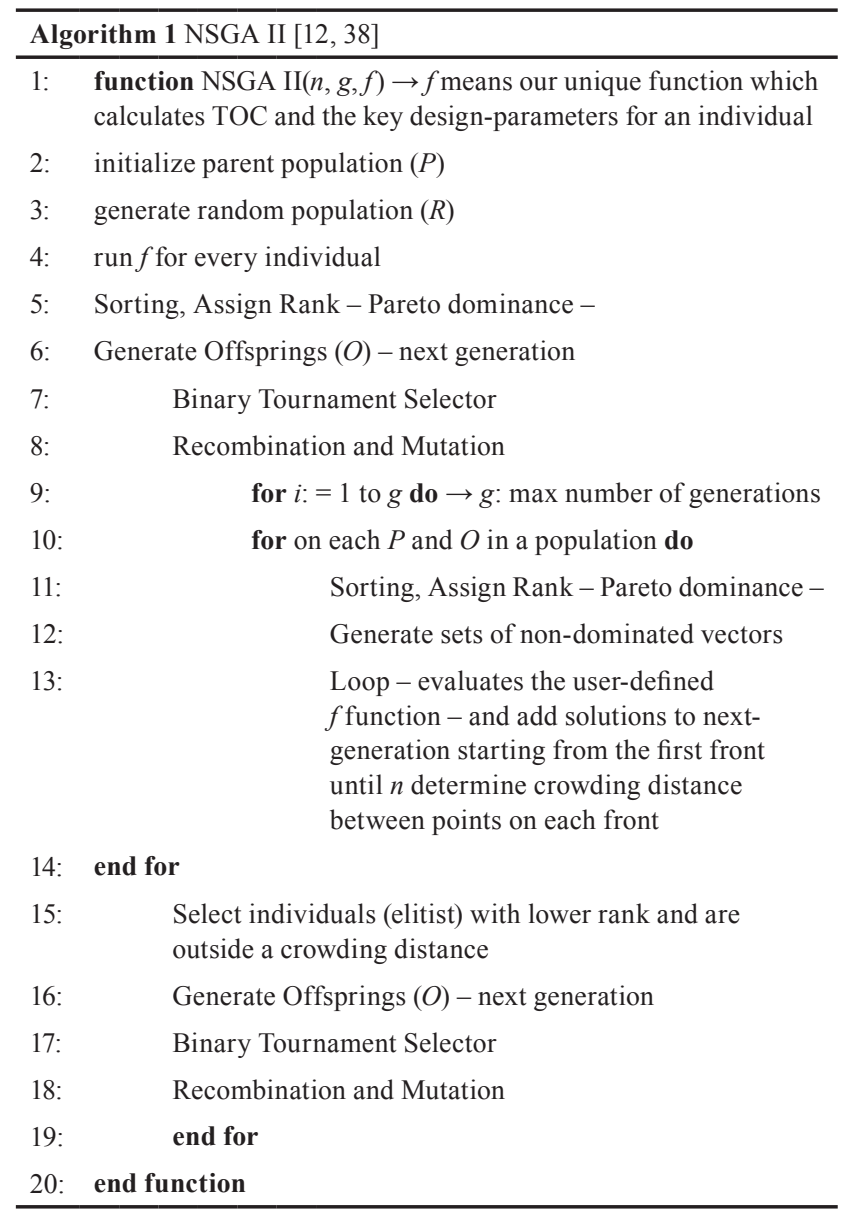

- $F_{\text {RMSE }}=\sqrt{\sum_{i=1}^{n}\left(P_{i}-M_{i}\right)^{2} / n}$

$F_{R^{2}}=1-\frac{\sum_{i=1}^{n}\left(P_{i}-M_{i}\right)^{2}}{\sum_{i=1}^{n}\left(P_{i}-A_{i}\right)^{2}}$

- $F_{\text {MAPE }}=\frac{\sum_{i=1}^{n}\left(A_{i}-P_{i}\right) / P_{i}}{n}$

- $F_{A}=n \times \log \left(\left(P_{i}-M_{i}\right)^{2}\right)+2 k$

where $P_{i}, M_{i}$ and $A_{i}$ are the predicted, measured and the averaged values, $n$ denote the sample size and $k$ is the number of the fittest parameters. There is some advice in the literature that if the parameters remain unchanged after the last 5 iterations, the iteration should be stopped [21, 24]. Algorithm 1 is used in this paper.

\section{Results and discussion}

To benchmark the measurement methodology, three measurements were made with three realized insulation model circuits. These models were made from precious resistors and capacitors, with $N=1,2$ and 3 branches, respectively. The values of the $R$ and $C$ parameters are shown in Table 1.

These values were measured by Agilent 4339B high resistance meter and Wayne Kerr 6430A component analyzer. Then, the Voltage Responses of model circuits were measured by the EVR equipment, which was developed at the Deapartment. The charging voltage and the charging time were $1000 \mathrm{~V}$ and $4000 \mathrm{~s}$, respectively. Due to the measurement uncertainty, the Quadratic Programming based methodology provides different values for $R_{p i}-C_{p i}$ elements of the Debye model. To prove the existence of one global optimum of the problem the ideal voltage slope parameters were calculated by analytical method based on Eqs. (1)-(3). These values provided a good benchmark to make the optimization on a data set without measurement error and uncertainties. The optimal parameters of the searched $R$ and $C$ values were calculated by two different methods. The first of them used the Ārtap and a built-in NSGA-II function. The used code and the project file can be downloaded from the project page of Ārtap, it is included in the package of [39]. The other calculation was made by the built-in optimizer of MATLAB, the GlobalSearch function [29]. This optimizer uses a Quadratic Programming solver, which starts from multiple points. The results of return voltage slopes are presented in Tables 2-4. Due to these properties, this metaheuristic solver is a very strong tool for this type of problem. The optimized parameters approximate well the original curvature. The fitted and the measured curves of slopes of return voltages as a function of shorting time are plotted in Fig. 4 in case of $N=3$.

In the case of $N=1$, both the NSGA-II and the GlobalSearch based calculations gave back the expected values with minimal calculation error. It can be seen that both solutions approximated well in the case of $N=2$ branches. The calculated values of each $R$ and $C$ parameters by NSGA-II and the GlobalSearch are significantly different in the $N=3$ case (case 3 ). This happened because

Table 1 The measured values of the $R$ and $C$ parameters.

\begin{tabular}{lccc}
\hline Parameter & $N=1$ & $N=2$ & $N=3$ \\
\hline$R_{0}[\mathrm{G} \Omega]$ & 5.87 & 5.87 & 5.87 \\
$R_{1}[\mathrm{G} \Omega]$ & 2.1663 & 2.1663 & 2.1663 \\
$R_{2}[\mathrm{G} \Omega]$ & - & 1.7806 & 1.7806 \\
$R_{3}[\mathrm{G} \Omega]$ & - & - & 3.0078 \\
$C_{0}[\mathrm{nF}]$ & 9.627 & 9.627 & 9.627 \\
$C_{1}[\mathrm{nF}]$ & 19.589 & 10.236 & 10.236 \\
$C_{2}[\mathrm{nF}]$ & - & 9.4081 & 9.4081 \\
$C_{3}[\mathrm{nF}]$ & & & 46.2880 \\
\hline
\end{tabular}


Table 2 The calculated voltage slopes based on analytical $(a)$ and optimised values of $R C$ parameters by GlobalSearch $(g)$ and NSGA-II $(n)$ in the case of $N=1$

\begin{tabular}{lccc}
\hline $\begin{array}{l}\text { Shorting time } \\
t(\mathrm{sec})\end{array}$ & \multicolumn{3}{c}{ Slopes of return voltages $(\mathrm{V} / \mathrm{s})$} \\
\hline 1 & 46.83347 & $S_{g}$ & $S_{n}$ \\
2 & 45.74274 & 45.73346 & 47.76 \\
4 & 43.6369 & 43.6369 & 46.458 \\
6 & 41.62801 & 41.628 & 43.153 \\
8 & 39.7116 & 39.7116 & 41.717 \\
10 & 37.88341 & 37.88341 & 39.697 \\
15 & 33.67273 & 33.67274 & 37.662 \\
20 & 29.93007 & 29.93008 & 33.207 \\
30 & 23.64647 & 23.64648 & 29.496 \\
50 & 14.75991 & 14.75992 & 14.522 \\
75 & 8.188986 & 8.188999 & 8.029 \\
100 & 4.543356 & 4.543365 & 4.479 \\
150 & 1.398524 & 1.398528 & 1.429 \\
200 & 0.43049 & 0.430492 & 0.454 \\
300 & 0.04079 & 0.04079 & 0.073 \\
500 & 0.000366 & 0.000366 & 0.001 \\
\hline
\end{tabular}

Table 3 The calculated voltage slopes based on analytical $(a)$ and optimised values of $R C$ parameters by GlobalSearch $(g)$ and NSGA-II $(n)$ in the case of $N=2$

\begin{tabular}{lccc}
\hline $\begin{array}{l}\text { Shorting time } \\
t(\mathrm{sec})\end{array}$ & \multicolumn{3}{c}{ Slopes of return voltages $(\mathrm{V} / \mathrm{s})$} \\
\hline 1 & 100.7923 & $S_{g}$ & $S_{n}$ \\
2 & 95.58673 & 95.58672 & 98.786 \\
4 & 85.98188 & 85.98188 & 94.485 \\
6 & 77.35857 & 77.35858 & 75.633 \\
8 & 69.6149 & 69.61492 & 68.024 \\
10 & 62.65971 & 62.65974 & 60.888 \\
15 & 48.20685 & 48.20688 & 46.472 \\
20 & 37.13707 & 37.13709 & 35.956 \\
30 & 22.12758 & 22.12759 & 21.456 \\
50 & 7.979435 & 7.979439 & 7.912 \\
75 & 2.29236 & 2.292368 & 2.318 \\
100 & 0.676797 & 0.676805 & 0.72 \\
150 & 0.062897 & 0.0629 & 0.069 \\
200 & 0.006189 & 0.006189 & 0.014 \\
300 & $6.49 \mathrm{E}-05$ & $6.49 \mathrm{E}-05$ & 0.026 \\
500 & $7.75 \mathrm{E}-09$ & $7.75 \mathrm{E}-09$ & 0.001 \\
\hline
\end{tabular}

of the optimized parameters are in the exponent of the objective function. Hence the objective function is relative flat consequently a small error in the precision of the parameter determination can produce a significantly high error. To present the problem difficulty the error function of objective function is calculated assuming the $R_{p i}$ and
Table 4 The calculated voltage slopes based on analytical $(a)$ and optimised values of $R C$ parameters by GlobalSearch $(g)$ and NSGA-II $(n)$ in the case of $N=3$

\begin{tabular}{lccc}
\hline $\begin{array}{l}\text { Shorting time } \\
t(\mathrm{sec})\end{array}$ & \multicolumn{3}{c}{ Slopes of return voltages (V/s) } \\
\hline 1 & 135.0715 & $S_{a}$ & $S_{n}$ \\
2 & 129.6215 & 129.0709 & 133.529 \\
4 & 119.5329 & 119.5332 & 128.043 \\
6 & 110.4325 & 110.433 & 117.659 \\
8 & 102.2183 & 102.2187 & 108.684 \\
10 & 94.79905 & 94.79937 & 92.426 \\
15 & 79.21414 & 79.21396 & 76.645 \\
20 & 67.05153 & 67.05094 & 65.032 \\
30 & 49.96951 & 49.96882 & 48.625 \\
50 & 32.09568 & 32.09618 & 31.257 \\
75 & 22.44427 & 22.44511 & 21.111 \\
100 & 17.51621 & 17.51614 & 16.426 \\
150 & 11.8215 & 11.82052 & 11.051 \\
200 & 8.217025 & 8.216676 & 7.65 \\
300 & 4.003668 & 4.004454 & 3.718 \\
500 & 0.951871 & 0.952578 & 0.89 \\
\hline
\end{tabular}

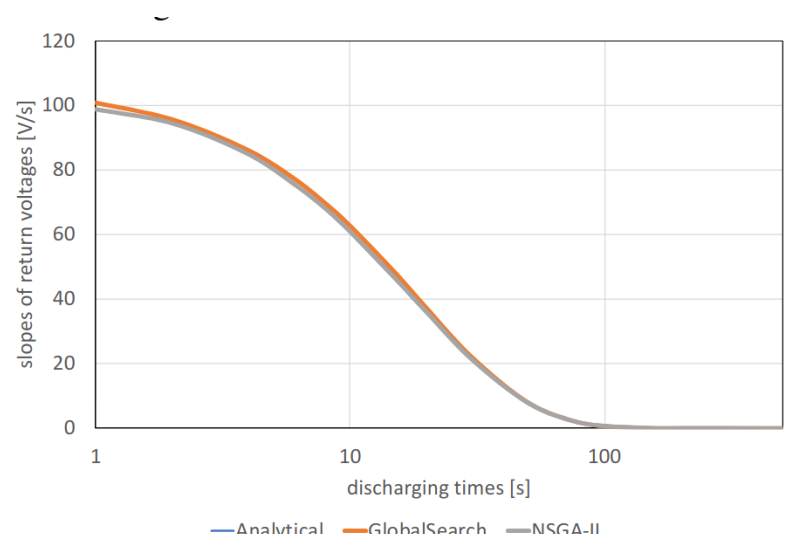

Fig. 4 The comparison of the model results and the fitted parameters in the case of $N=3$.

$C_{p i}$ values can be in range $1 \ldots 30 \mathrm{G} \Omega$ and $\mathrm{nF}$, repsctively (Fig. 5). The optimal values are represented by the darkest red region in the figure.

As the figures shows relative high variation in paremeters results in small change in error function therefore, the optimizated parameters of Debye elements are subject to high uncertainty.

The Quadratic Programming based methodology provides better results because it uses a Newton solver or a LevenbergMarquardt based solver to find the exact function parameters. The solution of the NSGA-II based methodology can be improved similarly if a Newton-solver started from the found parameters and then they are refined similarly [23]. 


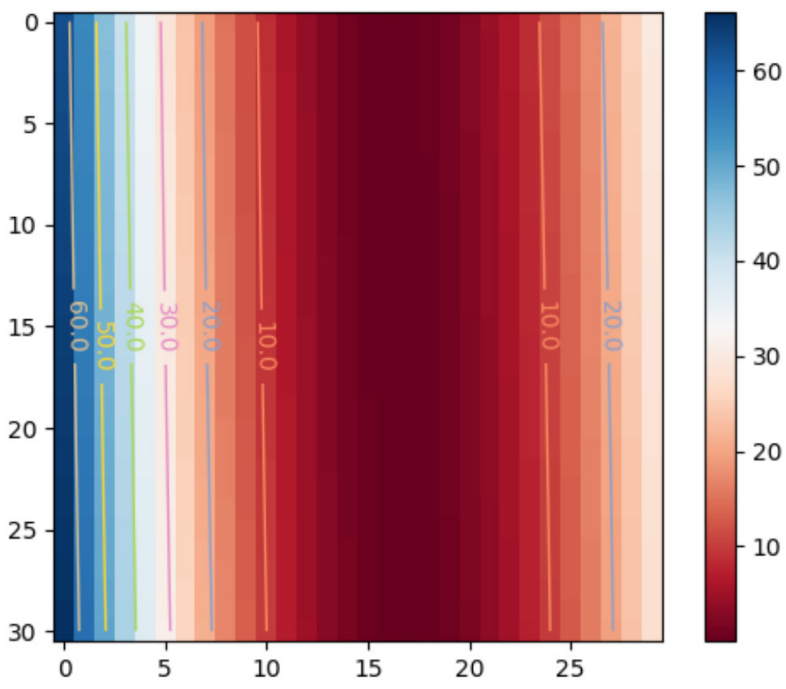

Fig. 5 The objective function error dependence on $R$ and $C$ parameters

\section{References}

[1] Mustafa, E., Afia, R. S. A., Tamus, Z. Á. "Condition Monitoring Uncertainties and Thermal - Radiation Multistress Accelerated Aging Tests for Nuclear Power Plant Cables: A Review", Periodica Polytechnica Electrical Engineering and Computer Science, 64(1), pp. 20-32, 2020.

https://doi.org/10.3311/PPee.14038

[2] Cselkó, R. "Repetition Rate of Partial Discharges in Low Voltage Cables", Periodica Polytechnica Electrical Engineering and Computer Science, 64(1), pp. 33-39, 2020. https://doi.org/10.3311/PPee.14434

[3] Zaengl, W. S. "Applications of dielectric spectroscopy in time and frequency domain for HV power equipment", IEEE Electrical Insulation Magazine, 19(6), pp. 9-22, 2003. https://doi.org/10.1109/MEI.2003.1266348

[4] Németh, E. "Measuring voltage response: a non-destructive diagnostic test method of HV insulation", IEE Proceedings-Science, Measurement and Technology, 146(5), pp. 249-252, 1999. https://doi.org/10.1049/ip-smt:19990651

[5] Tamus, Z. Á., Csányi, G. M. "Modeling of insulations by the results of voltage response measurement", presented at The 19th International Symposium on High Voltage Engineering, Pilsen, Czech Republik, Aug., 23-28, 2015.

[6] Tamus, Z. Á. "Combination of voltage response method with non-contact electrostatic voltage measurement to determine the dielectric response of insulating materials", Journal of Physics: Conference Series, 1322, Paper number: 012042, 2019. https://doi.org/10.1088/1742-6596/1322/1/012042

[7] Csanyi, G. M., Tamus, Z. Á. "Investigation of dielectric properties of mixed pilc and xlpE cable insulation by the extended Voltage response method", In: 2017 6th International Youth Conference on Energy (IYCE), Budapest, Hungary, 2017, pp. 1-4. https://doi.org/10.1109/IYCE.2017.8003702

\section{Conclusion}

The proposed paper has shown a novel experimental benchmark to determine the Debye representation of the insulating model. The parameterization is based on measurement results of Extended Voltage Response (EVR) on model dielectrics. As the results shows the proposed data and fitting problem can be used for testing the novel genetic, evolutionary algorithms, where benchmarking is an important and challenging problem. It can be seen from the results that this problem can be computationally hard and important to select a good metaheuristic solver to calculate the optimal $R$ and $C$ parameters of the Debye model.

[8] Mustafa, E., Tamus, Z. Á., Afia, R. S. A., Asipuela, A. "Thermal Degradation and Condition Monitoring of Low Voltage Power Cables in Nuclear Power Industry", In: Camarinha-Matos, L. M., Almeida, R., Oliveira, J. (eds.) Technological Innovation for Industry and Service Systems, vol. 553, Springer, Cham, Switzerland, 2019, pp. 405-413. https://doi.org/10.1007/978-3-030-17771-3_35

[9] Tóth, B. "Dual-mixed variational formulation and $h p$ finite element method for axisymmetric shell problems in elastodynamics", Proceedings in Applied Mathematics and Mechanics, 13(1), pp. 93-94, 2013. https://doi.org/10.1002/pamm.201310042

[10] Karban, P., Pánek, D., Orosz, T., Petrášova, I., Doležel, I. "Fem based robust design optimization with Agros and Ārtap", Computers \& Mathematics with Applications, 2020. https://doi.org/10.1016/j.camwa.2020.02.010

[11] Pánek, D., Karban, P., Orosz, T., Doležel, I. "Comparison of simplified techniques for solving selected coupled electro-heat problems", COMPEL-The international journal for computation and mathematics in electrical and electronicengineering, 39(1), pp. 220-230, 2020. https://doi.org/10.1108/COMPEL-06-2019-0244

[12] Pánek, D., Orosz, T., Karban, P. "Artap: Robust Design Optimization Framework for Engineering Applications", In: 2019 Third International Conference on Intelligent Computing in Data Sciences (ICDS), Marrakech, Morocco, 2019, pp. 1-6. https://doi.org/10.1109/ICDS47004.2019.8942318

[13] Orosz, T., Pánek, D., Karban, P. "Fem Based Preliminary Design Optimization in Case of Large Power Transformers", Applied Sciences, 10(4), Article number: 1361, 2020. https://doi.org/10.3390/app10041361 
[14] Cherif, B. D. E., Bendiabdellah, A., Bendjebbar, M., Tamer, A. "Neural Network Based Fault Diagnosis of Three Phase Inverter Fed Vector Control Induction Motor", Periodica Polytechnica Electrical Engineering and Computer Science, 63(4), pp. 295-305, 2019.

https://doi.org/10.3311/PPee.14315

[15] Henning, C., Schmid, A., Hecht, S., Harre, K., Bauer, R. "Applicability of Different Bio-based Polymers for Wiring Boards", Periodica Polytechnica Electrical Engineering and Computer Science, 63(1), pp. 1-8, 2019. https://doi.org/10.3311/PPee.13431

[16] Aissat, A., Guendoud, T. "Identification et Optimisation par Algorithmes Génétiques des Paramètres d'un Modèle de l'Hystérésis Magnétique" (Identification and Optimization by Genetic Algorithms of the Parameters of a Magnetic Hysteresis Model), PhD Thesis, Université Mouloud Mammeri, 2011. [oline] Available at: https://dl.ummto.dz/handle/ummto/9060 [Accessed: 15 August 2020] (in French)

[17] Quondam Antonio, S., Faba, A., Carlotti, G., Cardelli, E. "Vector hysteresis model identification for iron-silicon thin films from micromagnetic simulations", Physica B: Condensed Matter, 486, pp. $97-100,2016$. https://doi.org/10.1016/j.physb.2015.09.028

[18] Hergli, K., Marouani, H., Zidi, M., Fouad, Y., Elshazly, M. "Identification of Preisach hysteresis model parameters using genetic algorithms", Journal of King Saud University-Science, 31(4), pp. 746-752, 2019.

https://doi.org/10.1016/j.jksus.2017.11.005

[19] Abdelhadi, B., Benoudjit, A., Nait-Said, N. "Application of genetic algorithm with a novel adaptive scheme for the identification of induction machine parameters", IEEE Transactions on Energy Conversion, 20(2), pp. 284-291, 2005. https://doi.org/10.1109/TEC.2004.841508

[20] Lara-Ramirez, J. E., Garcia-Capulin, C. H., Estudillo-Ayala, M. J., Avina-Cervantes, J. G., Sanchez-Yanez, R. E., Rostro-Gonzalez, H. "Parallel Hierarchical Genetic Algorithm for Scattered Data Fitting through B-Splines", Applied Sciences, 9(11), Article number: 2336, 2019 .

https://doi.org/10.3390/app9112336

[21] Karr, C. L., Weck, B., Massart, D. L., Vankeerberghen, P. "Least Median Squares Curve Fitting Using a Genetic Algorithm", Engineering Applications of Artificial Intelligence, 8(2), pp. 177-189, 1995.

https://doi.org/10.1016/0952-1976(94)00064-T

[22] Sabsch, T., Braune, C., Dockhorn, A., Kruse, R. "Using a multiobjective genetic algorithm for curve approximation", In: 2017 IEEE Symposium Series on Computational Intelligence (SSCI), Honolulu, HI, USA, 2017, pp. 1-6. https://doi.org/10.1109/SSCI.2017.8285179

[23] Yang, F., Du, L., Yang, L., Wei, C., Wang, Y., Ran, L., He, P. "A Parameterization Approach for the Dielectric Response Model of Oil Paper Insulation Using FDS Measurements", Energies, 11(3), Article number: 622, 2018.

https://doi.org/10.3390/en11030622
[24] Zhang, Y., Niu, J., Na, S. "A Novel Nonlinear Function Fitting Model Based on FOA and GRNN", Mathematical Problems in Engineering, 2019, Article ID: 2697317, 2019. https://doi.org/10.1155/2019/2697317

[25] Gallagher, K., Sambridge, M. "Genetic Algorithms: A Powerful Tool for Large-scale Nonlinear Optimization Problems", Computers \& Geosciences, 20(7-8), pp. 1229-1236, 1994. https://doi.org/10.1016/0098-3004(94)90072-8

[26] Magnani, A., Boyd, S. P. "Convex piecewise linear fitting", Optimization and Engineering, 10(1), pp. 1-17, 2009. https://doi.org/10.1007/s11081-008-9045-3

[27] Boyd, S. P., Vandenberghe, L. "Convex optimization", Cambridge University Press, Cambridge, UK, 2004.

[28] Boyd, S., Kim, S.-J., Vandenberghe, L., Hassibi, A. "A tutorial on geometric programming", Optimization and Engineering, 8(1), pp. $67-127,2007$.

https://doi.org/10.1007/s11081-007-9001-7

[29] Ugray, Z., Lasdon, L., Plummer, J., Glover, F., Kelly, J., Martí, R. "Scatter Search and Local NLP Solvers: A Multistart Framework for Global Optimization", INFORMS Journal on Computing, 19(3), pp. 328-340, 2007. https://doi.org/10.1287/ijoc.1060.0175

[30] Orosz, T., Borbély, B., Tamus, Z. Á. "Performance Comparison of Multi-Design Method and Meta-Heuristic Methods for Optimal Preliminary Design of Core-Form Power Transformers", Periodica Polytechnica Electrical Engineering and Computer Science, 61(1), pp. 69-76, 2017. https://doi.org/10.3311/PPee.10207

[31] Orosz, T., Sleisz, Á., Tamus, Z. Á. "Metaheuristic Optimization Preliminary Design Process of Core-Form Autotransformers", IEEE Transactions on Magnetics, 52(4), pp. 1-10, 2016. https://doi.org/10.1109/TMAG.2015.2496905

[32] VanderNoot, T. J., Abrahams, I. "The use of genetic algorithms in the non-linear regression of immittance data", Journal of Electroanalytical Chemistry, 448(1), pp. 17-23, 1998. https://doi.org/10.1016/S0022-0728(97)00593-7

[33] Orosz, T. "Evolution and Modern Approaches of the Power Transformer Cost Optimization Methods", Periodica Polytechnica Electrical Engineering and Computer Science, 63(1), pp. 37-50, 2019. https://doi.org/10.3311/PPee.13000

[34] Orosz, T., Sleisz, Á., Vajda, I. "Core-form transformer design optimization with branch and bound search and geometric programming", In: 2014 55th International Scientific Conference on Power and Electrical Engineering of Riga Technical University (RTUCON), Riga, Latvia, 2014, pp. 17-21. https://doi.org/10.1109/RTUCON.2014.6998194

[35] Ho, Y. C., Pepyne, D. L. "Simple Explanation of the No-FreeLunch Theorem and Its Implications", Journal of optimization theory and applications, 115(3), pp. 549-570, 2002. https://doi.org/10.1023/A:1021251113462

[36] Deb, K., Pratap, A., Agarwal, S., Meyarivan, T. "A fast and elitist multiobjective genetic algorithm: NSGA-II", IEEE Transactions on Evolutionary Computation, 6(2), pp. 182-197, 2002. https://doi.org/10.1109/4235.996017 
[37] Padhye, N., Deb, K. "Multi-objective optimization and multi-criteria decision making in SLS using evolutionary approaches", Rapid Prototyping Journal, 17(6), pp. 458-478, 2011. https://doi.org/10.1108/13552541111184198

[38] Deb, K., Agrawal, S., Pratap, A., Meyarivan, T. "A Fast Elitist Non-dominated Sorting Genetic Algorithm for Multi-objective Optimization: NSGA-II", In: Schoenauer, M, Deb, K., Rudolph, G., Yao, X., Lutton, E., Merelo, J. J., Schwefel, H.-P. (eds.) Parallel Problem Solving from Nature - PPSN VI, vol. 1917, Springer, Berlin, Heidelberg, Germany, 2000, pp. 849-858.
[39] Pánek, D., Orosz, T., Karban, P. "Ārtap: artap 2020.4.2.1", [online] Available at: https://pypi.org/project/artap/2020.4.2.1/ [Accessed: 08 April 2020] 